\title{
Preventive effect of fermented brown rice and rice bran on $N$-methyl- $N$ '-nitro- $N$-nitrosoguanidine-induced gastric carcinogenesis in rats
}

\author{
HIROYUKI TOMITA $^{1,2}$, TOSHIYA KUNO ${ }^{1,3}$, YASUHIRO YAMADA ${ }^{1}$, TAKERU OYAMA $^{1}$, \\ NAMI ASANO ${ }^{1}$, YASUO MIYAZAKI ${ }^{1}$, SEIJI BABA ${ }^{1}$, AYAKO TAGUCHI $^{1}$, AKIRA HARA $^{1}$, \\ TERUAKI IWASAKI ${ }^{4}$, HIROSHI KOBAYASHI ${ }^{5}$ and HIDEKI MORI ${ }^{1}$
}

\begin{abstract}
${ }^{1}$ Departments of Tumor Pathology and ${ }^{2}$ Oncologic Surgery, Gifu University Graduate School of Medicine, 1-1 Yanagido, Gifu 501-1194; ${ }^{3}$ Onco-Pathology, Department of Pathology and Host-Defence, Faculty of Medicine, Kagawa University, 1750-1 Ikenobe, Miki-cho, Kita-gun, Kagawa 761-0793; ${ }^{4}$ Genmai Koso Co., Ltd., Nishi 1-chome, Kita-ku, Sapporo 001-0012; ${ }^{5}$ Sapporo Cancer Seminar, 6 Odori-Nishi, Chuo-ku, Sapporo 064-0820, Japan
\end{abstract}

Received August 16, 2007; Accepted September 28, 2007

\begin{abstract}
A number of possible preventive agents for cancers in different organs have been reported, however, little information is available regarding the effective agents for the development of gastric cancers. The rice components are known to be effective for the prevention of the development of cancers. Our group has demonstrated that fermented brown rice by Aspergillus Orzae (FBRA) has chemopreventive potentials in several organs. In this study, we investigated the modifying effects of FBRA exposed during the initiation or post-initiation phase of $N$-methyl- $N$ '-nitro- $N$-nitrosoguanidine (MNNG)-induced gastric carcinogenesis in rats. Five-weekold male ACI rats were divided into 7 groups. Groups 1-5 were given oral administration of MNNG (100 mg/l in distilled water) for 24 weeks starting at 6 weeks of age. Groups 2 and 3 were fed a diet containing 5 and 10\% FBRA during the initiation phase, respectively, whereas groups 4 and 5 were fed these diets during the post-initiation phase. Group 6 was given a diet containing 10\% FBRA throughout the experiment. Group 7 was kept on the basal diet alone and served as an untreated control. Rats were sacrificed at 52 weeks after the start, and the epithelium of the stomach was investigated in detail. Incidence and multiplicity of gastric proliferative lesions of group 1 (MNNG alone) were $61 \%$ and $1.67 \pm 1.57 /$ rat, respectively. Those of group $5(25 \%, 0.35 \pm 0.67)$ which were given FBRA at a dose of $10 \%$ during the post-initiation phase were significantly less than those of group 1 . Furthermore, the same group expressed a significantly
\end{abstract}

Correspondence to: Dr Hiroyuki Tomita, Department of Tumor Pathology, Gifu University Graduate School of Medicine, 1-1 Yanagido, Gifu 501-1194, Japan

E-mail: j2111706@edu.gifu-u.ac.jp

Key words: fermented brown rice, rice bran, gastric cancer decreased Ki67-labeling index in the non-lesional gastric epithelium when compared to that of group 1 . These results indicate that FBRA inhibits MNNG-induced development of gastric tumors by administration during the post-initiation phase in rats. FBRA is regarded as a promising dietary agent for the prevention of human gastric cancer.

\section{Introduction}

Gastric cancer is the fourth most common cancer and the second most common cause of cancer deaths worldwide $(1,2)$. Notwithstanding the global declining incidence of gastric cancer, mortality is still rising in Asian countries. Countries with a particularly high incidence of this disease include Japan, China and Korea. Most epidemiological studies show that a high intake of smoked, salted and nitrated foods, carbohydrates and a low intake of fruits, vegetables and milk significantly increase the risk of gastric cancer (2-4). It is suggested that the dietary consumption plays an important role in the development of gastric cancer, and natural products in traditional diets exert protective activities against the development of gastric cancers.

Rice is one of the major cereal foods eaten as a staple food worldwide, especially in Asian countries. Rice seeds and rice germ contain fiber and several kinds of antioxidants, such as ferulic acid (5), phytic acid (6), tocopherols and oryzanols (7). Among them, fiber $(8)$ and ferulic acid $(9,10)$ have been reported to prevent carcinogen-induced aerodigestive tract carcinogenesis in animal models. We have reported chemopreventive effects of rice germ itself or the compounds contained in the rice bran or germ against carcinogenesis in the large bowel or tongue of rodents $(11,12)$. Fermented brown rice by Aspergillus Orzae (FBRA) is a processed food prepared by fermenting brown rice and rice bran with Aspergillus Orzae. We previously reported that FBRA has inhibitory effects on the carcinogenesis of the colon, liver, esophagus and urinary bladder in rodents $(9,13-15)$. It is already known that FBRA has antioxidative activity and is 
regarded as an important mode of action of chemopreventive agents (16).

The method using $N$-methyl- $N$ '-nitro- $N$-nitrosoguanidine (MNNG) for the induction of stomach cancers in rodents is considered to be a good model of human gastric cancers $(17,18)$. In the present study, we examined the chemopreventive potential of FBRA on MNNG-induced gastric carcinogenesis in rats. In addition, we measured cell proliferation and apoptosis in the stomach epithelium for an understanding of functional mechanisms of FBRA.

\section{Materials and methods}

Animals, diets, carcinogen and FBRA. Male ACI rats, 4 weeks old, were purchased from Japan SLC, Inc. (Hamamatsu). After 1 week of quarantine, rats were transferred to the holding room under controlled conditions at $23 \pm 2^{\circ} \mathrm{C}$ temperature, $50 \pm 10 \%$ humidity, and a 12-h light/dark cycle. They were housed in wire cages ( 3 or 4 rats/cage). Powdered CE-2 (CLEA Japan, Inc., Tokyo) was used as the basal diet for the experiment. MNNG was obtained from Tokyo Chemical Inc. (Tokyo, Japan). The experimental diets were prepared by mixing 5 or $10 \%$ FBRA with CE- 2 diet. The diets were stored in a cold room $\left(4^{\circ} \mathrm{C}\right)$ and were freely available to the animals. FBRA was supplied by Genmai Koso Co., Ltd. (Sapporo, Japan). The manufacturing process of FBRA and its composition was reported previously (9).

Experimental procedure. The experimental design was approved by the Institutional Ethics Review Committee for animal experiments at the Gifu University. A total of 147 rats were randomized into 7 groups as shown in Fig. 1. At 6 weeks of age, rats in groups 1-5 received MNNG in drinking water for 24 weeks. MNNG (Tokyo Chemical) was dissolved in distilled water at a concentration of $100 \mathrm{mg} / \mathrm{l}$ and freshly prepared thrice per week for administration in drinking water in light-shielded bottles ad libitum, according to the protocol described in previous reports (19-21). Rats in groups 2 and 3 were given a diet containing 5 and 10\% FBRA for 26 weeks, respectively, starting one week before MNNG exposure until the end of week 26. They were switched to and maintained on the basal diet until termination. Starting 1 week after the cessation of MNNG treatment, rats in groups 4 and 5 were fed a diet mixed with 5 and $10 \%$ FBRA, respectively, which continued until termination. Rats in group 6 were fed a diet containing $10 \%$ FBRA during the experiment. Rats in group 7 were given the basal diet and tap water throughout the experiment and served as controls. All rats were carefully inspected daily, and consumption of the experimental diets mixed with the test compound was recorded to estimate dietary intake. Fifty-two weeks after it began, the experiment was terminated.

Tissue preparation and histopathological examination. At the end of the experiment, all rats were sacrificed to evaluate the frequency of preneoplastic and neoplastic lesions in the stomach. During the autopsy, the stomach of all the rats were inflated with $10 \%$ buffered formalin, fixed overnight in $10 \%$ buffered formalin, bisected longitudinally, inspected for gross lesions, then embedded in paraffin for a histopathological evaluation on hematoxylin and eosin (H\&E)-stained sections. The defining characteristics for atypical hyperplasia and adenocarcinoma were adapted from previous literature (22). Atypical hyperplasia were characterized by glandular proliferation with mild structural and cellular atypia, intramucosal adenocarcinomas by excessive glandular proliferation with pronounced structural and cellular atypia within the mucosa, and invasive adenocarcinomas by cellular atypia and atypical glandular structures with invading at least the submucosa. Other macroscopically abnormal organs were examined histologically. The liver and kidney were fixed with $10 \%$ formalin, embedded in paraffin blocks and processed routinely for a histopathological examination.

Immunohistochemistry. The avidin-biotin peroxidase complex $(\mathrm{ABC})$ technique was used for immunohistochemical studies. Five- $\mu \mathrm{m}$ thick sections were cut, deparaffinized, rehydrated in PBS, placed in $10 \mathrm{mmol} / \mathrm{l}$ citrate buffer $(\mathrm{pH} \mathrm{6.0)}$ ), and heated in a $750-\mathrm{W}$ microwave four times for $6 \mathrm{~min}$. The endogenous peroxidase activity was blocked by incubation for $30 \mathrm{~min}$ in $0.3 \mathrm{H}_{2} \mathrm{O}_{2}$. After washing three times with PBS, the sections were preincubated with a normal blocking serum for $20 \mathrm{~min}$ at room temperature and then incubated with Ki67 (Dako, Carpinteria, CA, \#M7248; 1:200) and cleaved caspase-3 (Cell Signaling, Danvers, MA, \#9661; 1:200) overnight at $4^{\circ} \mathrm{C}$. Subsequently, the sections were incubated with biotinylated secondary antibodies (Vectastain ABC kit, Vector Laboratories) for $30 \mathrm{~min}$, followed by incubation with avidincoupled peroxidase (Vector Laboratories) for $30 \mathrm{~min}$. The sections were developed with 3,3V-diaminobenzidine (DAB) using Dako liquid DAB substrate-chromogen system (Dako Corp.) and were then counterstained with hematoxylin. The specificity of the binding was confirmed by omitting the primary antibody, and this staining was used as a negative control.

Cell proliferation and apoptosis analysis. Cell proliferation index in non-lesional lesions of the stomach was determined by immunochemistry with a Ki67 antibody. Cells undergoing apoptosis were determined by immunochemistry with activated cysteinyl aspartic acid-protease-3 (cleaved caspase-3) antibody. The percentage of Ki67- and cleaved caspase-3positive cells, i.e., the labeling index in gastric gland tissues were determined by two persons in a blinded manner regarding the conditions of the section. An average of 500 cells were counted for each section stained for the detection of either Ki67- or cleaved caspase-3-positive cells under x400 magnification at light microscopy.

Stastical analysis. Statistical analysis of the incidence of gastric lesions was performed using the Fisher's exact test. The data on the multiplicity of gastric lesions, Ki67 index and apoptosis index were examined by using the Student's t-test.

\section{Results}

General observation of experimental animals. Rats from groups 1-7 tolerated well the oral administration of MNNG. There were no significant differences in the mean intake of MNNG among groups 1-5. The mean intake of food was 


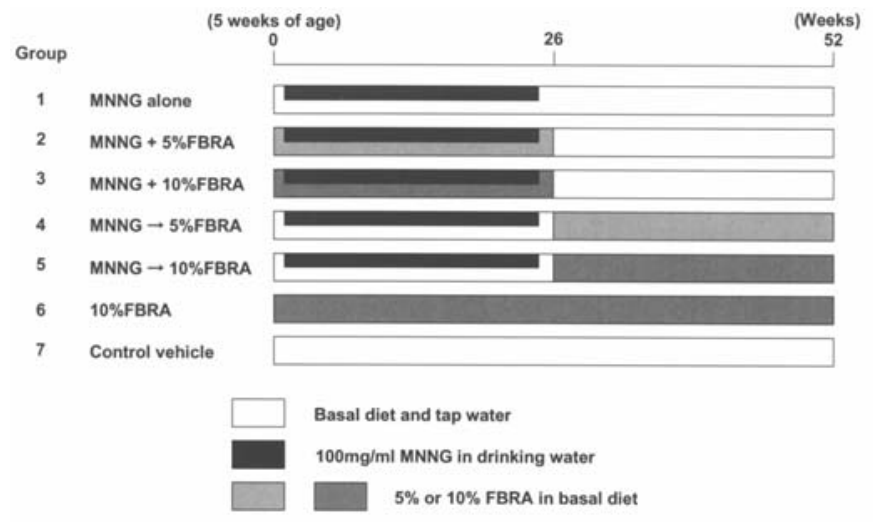

Figure 1. Experimental design. A total of 147 rats were used in the experiment. All rats were carefully autopsied at the time of their death, either after having been sacrificed because they had become moribund or at the end of the experiment (week 52).

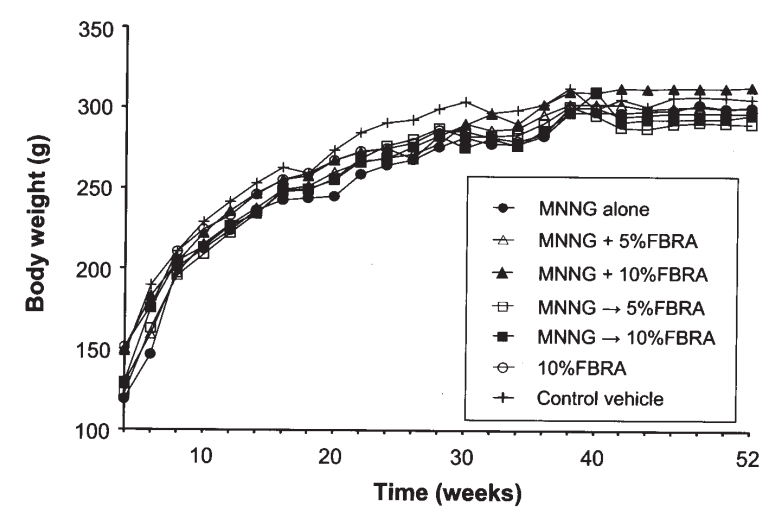

Figure 2. Body weight of animals in the different treatment groups during the experiment. There was no significant difference in body weight among the groups.

constant and similar among the different groups during the entire treatment period. There was no significant difference in body weight among all groups during the entire study period (Fig. 2). In addition, there were no marked differences in liver, or kidney weight among the groups (data not shown). There were no toxicopathological findings in the liver and kidney of any rat examined. There were in total 8 deaths during the study period, which were due to gastrointestinal cancer $(n=4)$ or non-digestive tract diseases $(n=4)$.

Effect of FBRA on glandular stomach carcinogenesis. The gastric tumors were solitary or multiple lesions and developed in the glandular stomach, predominantly in the antrum (Fig. 3). Histologically, these tumors were atypical hyperplasia or adenocarcinomas. In addition, several spindle sarcomas were developed in the glandular stomach. The incidence and multiplicity (number of tumors/rat) of gastric tumors are summarized in Tables I and II, respectively. Atypical hyperplasia was classified as a benign tumor, whereas adenocarcinoma and sarcoma were classified as malignancies. All of these lesions were defined as proliferative lesions in the glandular stomach. Gastric proliferative lesions were found only in groups 1-5. The incidence of total malignancies and
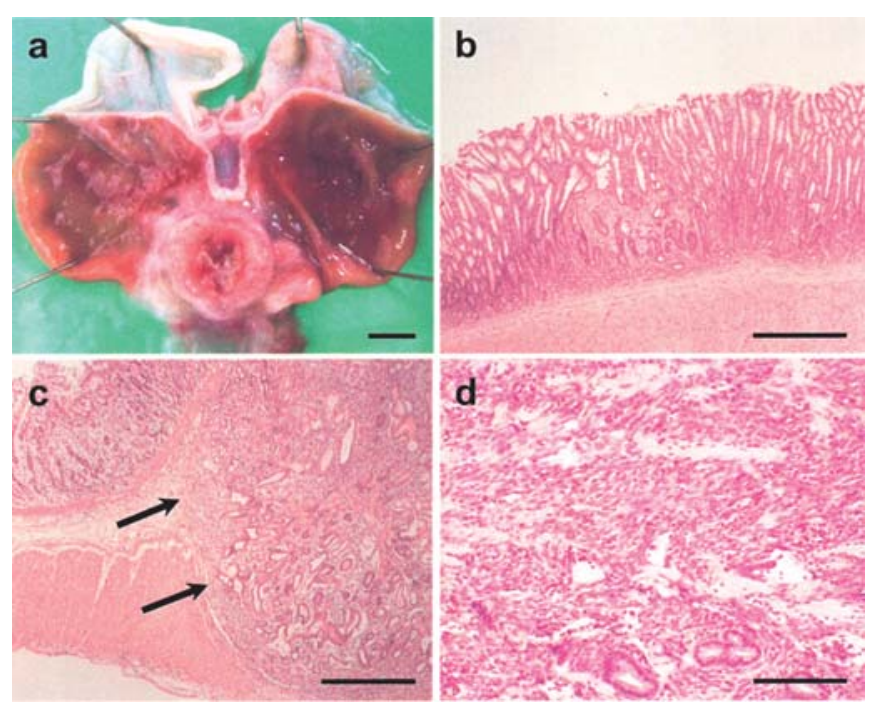

Figure 3. Macroscopic and microscopic appearance of MNNG-induced gastric tumors. (a) Representative macroscopic appearance of MNNG-induced tumor formation. Bar, $5 \mathrm{~mm}$. (b-d) H\&E staining of proliferative lesions in the stomach. (b) Atypical hyperplasia. Bar, $500 \mu \mathrm{m}$. (c) Invasive adenocarcinoma. Arrows indicate the invasion of cancer cells. Bar, $500 \mu \mathrm{m}$. (d) Spindle cell sarcoma. Bar, $100 \mu \mathrm{m}$.

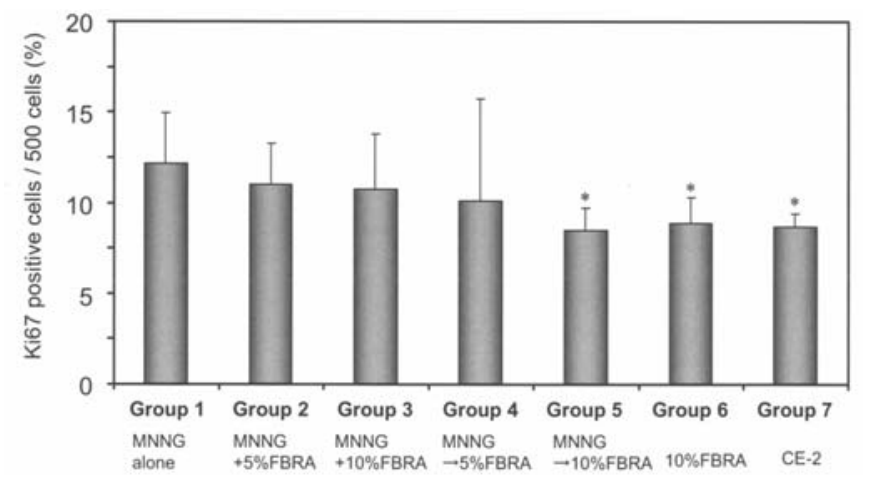

Figure 4. Effect of FBRA on the proliferation index in the non-lesional epithelium of the glandular stomach as detailed in Materials and methods. Columns, mean; Bars, SD; ${ }^{*} \mathrm{P}<0.05$ versus group 1 .

proliferative lesions in group 5 was significantly lower than that of group $1(\mathrm{P}<0.05)$. As shown in Table II, the mean number per animals (multiplicities) of total malignancies and proliferative lesions in group 5 was markedly decreased in comparison with that in group $1(\mathrm{P}<0.01)$. Furthermore, the number of atypical hyperplasias and invasive adenocarcinomas of group 5 were significantly smaller than that in group 1 $(\mathrm{P}<0.05)$. In addition, total number of malignancies in groups 4 and 5 were significantly smaller than that in group $1(\mathrm{P}<0.05)$.

Cell proliferation and apoptosis in the non-lesional gastric epithelium. The results of Ki67-labeling index in the nonlesional gastric epithelium of rats in each group are shown in Fig. 4. The Ki67-labeling index of group 5 was significantly smaller than that of group $1(\mathrm{P}<0.05)$. Similarly, the index of group 6 or 7 was smaller than that of group 1. Regarding apoptotic index, there were no statistically significant differences among the groups (data not shown). 
Table I. The incidence rate of glandular stomach proliferative lesions in rats.

\begin{tabular}{|c|c|c|c|c|c|c|c|c|c|}
\hline \multirow[b]{4}{*}{ Group } & \multirow[b]{4}{*}{ Treatment } & \multirow[b]{4}{*}{$\begin{array}{l}\text { Effective } \\
\text { no. of rats }\end{array}$} & \multicolumn{7}{|c|}{ No. of rats with proliferative lesions (\%) } \\
\hline & & & \multirow[b]{3}{*}{$\mathrm{AH}^{\mathrm{a}}$} & \multicolumn{5}{|c|}{ Malignancies } & \multirow[b]{3}{*}{$\begin{array}{c}\text { Total proliferative } \\
\text { lesions }\end{array}$} \\
\hline & & & & \multicolumn{3}{|c|}{$\mathrm{ADC}^{\mathrm{b}}$} & \multirow[b]{2}{*}{$\begin{array}{l}\text { Spindle } \\
\text { cell } \\
\text { sarcoma }\end{array}$} & \multirow[b]{2}{*}{ Total } & \\
\hline & & & & $\begin{array}{c}\text { Non- } \\
\text { invasive } \\
\text { ADC }\end{array}$ & $\begin{array}{l}\text { Invasive } \\
\text { ADC }\end{array}$ & Total & & & \\
\hline 1 & MNNG & 18 & $7(39)$ & $3(17)$ & $6(33)$ & $7(39)$ & $3(17)$ & $10(56)$ & $11(61)$ \\
\hline 2 & $\mathrm{MNNG}+5 \%$ FBRA & 21 & $7(33)$ & $2(10)$ & $4(19)$ & $5(24)$ & $1(5)$ & $5(24)$ & $8(38)$ \\
\hline 3 & MNNG + $10 \%$ FBRA & 20 & $5(25)$ & $1(5)$ & $3(15)$ & $3(15)$ & 0 & $3(15)$ & $7(35)$ \\
\hline 4 & $\mathrm{MNNG} \rightarrow 5 \% \mathrm{FBRA}$ & 20 & $3(15)$ & $1(5)$ & $3(15)$ & $4(20)$ & $1(5)$ & $5(25)$ & $6(30)$ \\
\hline 5 & $\mathrm{MNNG} \rightarrow 10 \% \mathrm{FBRA}$ & 20 & $2(10)$ & $1(5)$ & $3(15)$ & $4(20)$ & 0 & $4(20)^{\mathrm{c}}$ & $5(25)^{c}$ \\
\hline 6 & $10 \%$ FBRA alone & 20 & 0 & 0 & 0 & 0 & 0 & 0 & 0 \\
\hline 7 & Control vehicle & 20 & 0 & 0 & 0 & 0 & 0 & 0 & 0 \\
\hline
\end{tabular}

${ }^{\mathrm{a}} \mathrm{AH}$, atypical hyperplasia; ${ }^{\mathrm{b}} \mathrm{ADC}$, adenocarcinoma. ${ }^{\mathrm{C}} \mathrm{P}<0.05$ versus group 1.

Table II. Multiplicities of glandular stomach proliferative lesions in rats.

\begin{tabular}{|c|c|c|c|c|c|c|c|c|c|}
\hline \multirow[b]{4}{*}{ Group } & \multirow[b]{4}{*}{ Treatment } & \multirow[b]{4}{*}{$\begin{array}{l}\text { Effective } \\
\text { no. of rats }\end{array}$} & \multicolumn{7}{|c|}{ No. of gastric lesions per rat (mean \pm SD) } \\
\hline & & & \multirow[b]{3}{*}{$\mathrm{AH}^{\mathrm{a}}$} & \multicolumn{5}{|c|}{ Malignancies } & \multirow[b]{3}{*}{$\begin{array}{c}\text { Total proliferative } \\
\text { lesions }\end{array}$} \\
\hline & & & & \multicolumn{3}{|c|}{$\mathrm{ADC}^{\mathrm{b}}$} & \multirow[b]{2}{*}{$\begin{array}{c}\text { Spindle } \\
\text { cell } \\
\text { sarcoma }\end{array}$} & \multirow[b]{2}{*}{ Total } & \\
\hline & & & & $\begin{array}{c}\text { Non- } \\
\text { invasive } \\
\text { ADC }\end{array}$ & $\begin{array}{l}\text { Invasive } \\
\text { ADC }\end{array}$ & Total & & & \\
\hline 1 & MNNG & 18 & $0.56 \pm 0.78$ & $0.17 \pm 0.38$ & $0.78 \pm 1.22$ & $0.94 \pm 1.30$ & $0.17 \pm 0.38$ & $1.67 \pm 1.23$ & $1.67 \pm 1.57$ \\
\hline 2 & $\mathrm{MNNG}+5 \% \mathrm{FBRA}$ & 21 & $0.57 \pm 0.98$ & $0.14 \pm 0.4$ & $0.33 \pm 0.80$ & $0.48 \pm 1.17$ & $0.05 \pm 0.22$ & $0.52 \pm 1.21$ & $1.10 \pm 1.70$ \\
\hline 3 & MNNG+ $10 \%$ FBRA & 20 & $0.75 \pm 1.45$ & $0.05 \pm 0.22$ & $0.20 \pm 0.52$ & $0.25 \pm 0.64$ & 0 & $0.25 \pm 0.64$ & $1.00 \pm 1.65$ \\
\hline 4 & $\mathrm{MNNG} \rightarrow 5 \% \mathrm{FBRA}$ & 20 & $0.55 \pm 1.19$ & $0.05 \pm 0.22$ & $0.20 \pm 0.52$ & $0.25 \pm 0.55^{\mathrm{c}}$ & $0.05 \pm 0.22$ & $0.30 \pm 0.57^{\mathrm{c}}$ & $0.85 \pm 1.57$ \\
\hline 5 & $\mathrm{MNNG} \rightarrow 10 \% \mathrm{FBRA}$ & 20 & $0.15 \pm 0.49^{\mathrm{c}}$ & $0.05 \pm 0.22$ & $0.15 \pm 0.37^{\mathrm{c}}$ & $0.20 \pm 0.41^{\mathrm{c}}$ & 0 & $0.20 \pm 0.41^{\mathrm{d}}$ & $0.35 \pm 0.67^{d}$ \\
\hline 6 & $10 \%$ FBRA alone & 20 & 0 & 0 & 0 & 0 & 0 & 0 & 0 \\
\hline 7 & Control vehicle & 20 & 0 & 0 & 0 & 0 & 0 & 0 & 0 \\
\hline
\end{tabular}

${ }^{\mathrm{a}} \mathrm{AH}$, atypical hyperplasia; ${ }^{\mathrm{b}} \mathrm{ADC}$, adenocarcinoma.. ${ }^{\mathrm{c}} \mathrm{P}<0.05$ and ${ }^{\mathrm{d}} \mathrm{P}<0.01$ versus group 1 .

\section{Discussion}

This study was undertaken in order to evaluate the efficacy of FBRA on carcinogen-induced gastric tumorigenesis in rats. In this study, administration of $10 \%$ FBRA in the diet during the post-initiation phase inhibited MNNG-induced gastric carcinogenesis in the level of the total number of total proliferative lesions, including malignancies. Furthermore, the treatment of $10 \%$ FBRA in the post-initiation phase also reduced the development of stomach adenocarcinomas in a dose-dependent manner. This effect of FBRA on the gastric carcinogenesis is consistent with the results of our previous studies where FBRA suppressed the carcinogenesis of the colon, liver, esophagus or urinary bladder by its exposure during the post-initiation phase $(9,13-15)$.

Although the exact underlying mechanism for the suppressive effect of FBRA on gastric carcinogenesis is not clear, multiple constituents of FBRA are suggested to be related to the modifying effect. Antioxidants in FBRA such as ferulic acid or phytic acid may be one of the responsive agents. In fact, the presence of a high amount of ferulic acid in FBRA is confirmed (23). Previously, our group reported a protective effect of ferulic acid, a polyphenol on the tongue or colorectal carcinogenesis in rodents $(10,24)$. In another experiment, we also proved the suppressive effect of chlorogenic acid, a related antioxidative polyphenol, on $\mathrm{N}$ methyl-N-nitrosourea-induced gastric carcinogenesis in rats (25). It is also known that dihydroferulic acid or dihydrosinaptic acid contained in an unpolished rice vinegar possesses a potent radical scavenging activity (26). It is possible that the effect of FBRA is mediated through the action of several trace elements, including selenium which is biotransformed to an organic form during the fermentation of brown rice and rice bran (27-29).

In general, control of cell proliferation in the target organs for carcinogenesis is regarded as one of most important modes of action of chemopreventive agents (30). In this study, dietary exposure of FBRA significantly reduced Ki67-labeling 
indices in the non-lesional gastric epithelium. Thus, the inhibitory effect of FBRA against carcinogen-induced hyperproliferation of the cells in the gastric mucosa is suggested to relate to the suppressive effect on the gastric carcinogenesis. Unfortunately, in this experiment, no clear difference in the apoptotic indices was found among each group in the analysis of apoptosis for the non-lesional gastric epithelium. Nevertheless, it may still be possible that dietary exposure of FBRA over a long-term during the post-initiation phase excludes carcinogen-induced initiated cells in the gastric epithelium. Recently, Sakurai et al (23) reported that the inhibitory effect of FBRA against liver metastasis of cancer cells is caused by cytokine generating type 1 helper $\mathrm{T}$ celldominant immune state and activation of macrophages. Such an immunostimulatory effect generated by the exposure of FBRA may also be involved in the suppressive effect on the gastric carcinogenesis in rodents.

In conclusion, we demonstrated that the dietary administration of $10 \%$ FBRA during the post-initiation phase suppresses MNNG-induced tumor development in the stomach of rats. FBRA is considered to be a promising chemopreventive agent against the occurrence of human gastric cancers.

\section{Acknowledgements}

We thank Akihiro Hirata for helpful discussions. We thank Kyoko Takahashi, Ayako Suga and Yoshitaka Kinjyo for their technical assistance and animal care. We also thank Satomi Goto for the secretarial assistance. This study was supported by grants from the Ministry of Health, Labour and Welfare of Japan, and grants from the Ministry of Education, Culture, Sports, Science and Technology of Japan.

\section{References}

1. Correa P, Piazuelo MB and Camargo MC: The future of gastric cancer prevention. Gastric Cancer 7: 9-16, 2004.

2. Fox JG and Wang TC: Helicobacter pylori-not a good bug after all! N Engl J Med 345: 829-832, 2001.

3. Wu AH, Yang D and Pike MC: A meta-analysis of soyfoods and risk of stomach cancer: the problem of potential confounders. Cancer Epidemiol Biomarkers Prev 9: 1051-1058, 2000.

4. Howson CP, Hiyama T and Wynder EL: The decline in gastric cancer: epidemiology of an unplanned triumph. Epidemiol Rev 8: 1-27, 1986.

5. Taniguchi H, Hosoda A, Tsuno T, Maruta Y and Nomura E: Preparation of ferulic acid and its application for the synthesis of cancer chemopreventive agents. Anticancer Res 19: 3757-3761, 1999.

6. Graf E, Empson KL and Eaton JW: Phytic acid. A natural antioxidant. J Biol Chem 262: 11647-11650, 1987.

7. $\mathrm{Xu} \mathrm{Z}$, Hua N and Godber JS: Antioxidant activity of tocopherols, tocotrienols, and gamma-oryzanol components from rice bran against cholesterol oxidation accelerated by 2,2'-azobis(2methylpropionamidine) dihydrochloride. J Agric Food Chem 49: 2077-2081, 2001.

8. van Rensburg SJ, Hall JM and du Bruyn DB: Effects of various dietary staples on esophageal carcinogenesis induced in rats by subcutaneously administered N-nitrosomethylbenzylamine. J Natl Cancer Inst 75: 561-566, 1985.

9. Katyama M, Yoshimi N, Yamada Y, et al: Preventive effect of fermented brown rice and rice bran against colon carcinogenesis in male F344 rats. Oncol Rep 9: 817-822, 2002.

10. Kawabata K, Yamamoto T, Hara A, et al: Modifying effects of ferulic acid on azoxymethane-induced colon carcinogenesis in F344 rats. Cancer Lett 157: 15-21, 2000.
11. Kawabata K, Tanaka T, Murakami T, et al: Dietary prevention of azoxymethane-induced colon carcinogenesis with rice-germ in F344 rats. Carcinogenesis 20: 2109-2115, 1999.

12. Mori H, Kawabata K, Yoshimi N, et al: Chemopreventive effects of ferulic acid on oral and rice germ on large bowel carcinogenesis. Anticancer Res 19: 3775-3778, 1999.

13. Kuno T, Hirose Y, Yamada Y, et al: Chemoprevention of mouse urinary bladder carcinogenesis by fermented brown rice and rice bran. Oncol Rep 15: 533-538, 2006.

14. Kuno T, Hirose Y, Hata K, et al: Preventive effect of fermented brown rice and rice bran on $\mathrm{N}$-nitrosomethylbenzylamineinduced esophageal tumorigenesis in rats. Int $\mathrm{J}$ Oncol 25: 1809-1815, 2004.

15. Katayama M, Sugie S, Yoshimi N, et al: Preventive effect of fermented brown rice and rice bran on diethylnitrosoamine and phenobarbital-induced hepatocarcinogenesis in male F344 rats. Oncol Rep 10: 875-880, 2003.

16. Tazawa $K$, Naoko $F$ and Namikawa $H$ : Superoxide scavenging effect of fermented brown rice determined by ESR spin-tapping method (in Japanese). Food Style 3: 32-37, 1999.

17. Sugimura T and Fujimura S: Tumour production in glandular stomach of rat by $\mathrm{N}$-methyl-N'-nitro-N-nitrosoguanidine. Nature 216: 943-944, 1967.

18. Ohgaki H, Kusama K, Hasegawa H, Sato S, Takayama S and Sugimura T: Sequential histologic changes during gastric carcinogenesis induced by N-methyl-N'-nitro-N-nitrosoguanidine in susceptible ACI and resistant BUF rats. J Natl Cancer Inst 77: 747-755, 1986.

19. Hu PJ, Yu J, Zeng ZR, et al: Chemoprevention of gastric cancer by celecoxib in rats. Gut 53: 195-200, 2004.

20. Mei Y, Wei D and Liu J: Modulation effect of tea polyphenol toward N-methyl-N'-nitro-N-nitrosoguanidine-induced precancerous gastric lesion in rats. J Nutr Biochem 16: 172-177, 2005.

21. Sugie S, Okamoto K, Watanabe T, Tanaka T and Mori H: Suppressive effect of irsogladine maleate on N-methyl-N-nitro$\mathrm{N}$-nitrosoguanidine (MNNG)-initiated and glyoxal-promoted gastric carcinogenesis in rats. Toxicology 166: 53-61, 2001.

22. Ikezaki S, Nishikawa A, Furukawa F, et al: Chemopreventive effects of 24R,25-dihydroxyvitamin D3, a vitamin D3 derivative, on glandular stomach carcinogenesis induced in rats by Nmethyl-N'-nitro-N-nitrosoguanidine and sodium chloride. Cancer Res 56: 2767-2770, 1996.

23. Sakurai H, Choo M-K, Chino A, Iwasaki T, Kobayashi H and Saiki I: Antimetastatic and immunostimulatory properties of fermented brown rice. J Tradit Med 23: 112-116, 2006.

24. Tanaka T, Kojima T, Kawamori T, et al: Inhibition of 4nitroquinoline-1-oxide-induced rat tongue carcinogenesis by the naturally occurring plant phenolics caffeic, ellagic, chlorogenic and ferulic acids. Carcinogenesis 14: 1321-1325, 1993.

25. Shimizu M, Yoshimi N, Yamada Y, et al: Suppressive effects of chlorogenic acid on N-methyl-N-nitrosourea-induced glandular stomach carcinogenesis in male F344 rats. J Toxicol Sci 24: 433-439, 1999.

26. Shimoji Y, Tamura Y, Nakamura Y, et al: Isolation and identification of DPPH radical scavenging compounds in Kurosu (Japanese unpolished rice vinegar). J Agric Food Chem 50: 6501-6503, 2002.

27. Lanfear J, Fleming J, Walker M and Harrison P: Different patterns of regulation of the genes encoding the closely related $56 \mathrm{kDa}$ selenium- and acetaminophen-binding proteins in normal tissues and during carcinogenesis. Carcinogenesis 14: 335-340, 1993.

28. Aquino TM, Porta EA, Sablan HM and Dorado RD: Effects of selenium supplementation on hepatocarcinogenesis in rats. Nutr Cancer 7: 25-36, 1985.

29. LeBoeuf RA, Laishes BA and Hoekstra WG: Effects of dietary selenium concentration on the development of enzyme-altered liver foci and hepatocellular carcinoma induced by diethylnitrosamine or $\mathrm{N}$-acetylaminofluorene in rats. Cancer Res 45: 5489-5495, 1985.

30. Mori H, Niwa K, Zheng Q, Yamada Y, Sakata K and Yoshimi N: Cell proliferation in cancer prevention; effects of preventive agents on estrogen-related endometrial carcinogenesis model and on an in vitro model in human colorectal cells. Mutat Res 480-481: 201-207, 2001. 